\title{
The Implantation of Left Bundle Branch Area Pacing in Patients with and without Bundle Branch Block
}

\author{
Tian-Ping Chen, Xiao-Jun Shi, Dong-Yu Lu and Heng Zhang \\ Department of Cardiology, The First Affiliated Hospital of Bengbu Medical College, Bengbu, China
}

\begin{abstract}
Objective: To investigate the clinical safety and electrocardiogram (ECG) characteristics in patients with left bundle branch area pacing (LBBAP).

Study Design: Retrospective study.

Place and Duration of Study: Department of Cardiology, The First Affiliated Hospital of Bengbu Medical College, Bengbu, China, from May 2018 to January 2020.

Methodology: Patients scheduled for Left Bandle Branch Area Pacing (LBBAP), who were admitted due to bradycardia, had been prospectively recruited. The Medtronic 3830 pacing lead was first placed at the right ventricular (RV) side of the interventricular septum (IVS) with pacing parameters (pacing threshold, pacing impedance and sensing amplitude) and ECG characteristics [QRS morphology, paced QRS duration and stimulus to peak left ventricular activation time (Sti-LVAT)] measured, which was called the right ventricular septum pacing group (RVSP). Then the pacing lead was screwed towards the left ventricular (LV) side of the IVS; and the corresponding parameters and ECG characteristics were assessed, which was called LBBAP group.

Results: RVSP caused left bundle block (LBBB) morphology on ECG, while pacing at left bundle area led to right bundle branch block (RBBB) morphology, without remarkable difference in pacing threshold and pacing impedance. The sensing amplitude during LBBAP was significantly higher compared with RVSP $(p<0.05)$. QRS duration and Sti-LVAT were significantly shorter when paced on LBBAP compared with RVSP $(p<0.05)$. Patients with LBBB morphology in intrinsic rhythm showed the greatest reduction in paced QRS duration and Sti-LVAT compared to patients with RBBB morphology or no bundle branch block morphology $(p<0.001)$. There were no complications during pacemaker implantation and no adverse events observed during follow-up. The pacing parameters remained stable during the follow-up $(9.2 \pm 3.7$ months).

Conclusion: Compared with pacing on RVSP, patients with LBBAP showed RBBB morphology with significantly reduced QRS duration and LV Sti-LVAT under similar pacing parameters. LBBAP is safe and feasible and may be a promising strategy for patients with LBBB morphology who are indicated for ventricular pacing.
\end{abstract}

Key Words: Physiological pacing, Left bundle branch pacing, Right ventricular pacing, Left bundle branch block, Pacemaker.

How to cite this article: Chen TP, Shi XJ, Lu DY, Zhang H. The Implantation of Left Bundle Branch Area Pacing in Patients with and without Bundle Branch Block. J Coll Physicians Surg Pak 2021; 31(11):1268-1272.

\section{INTRODUCTION}

Sick sinus syndrome and complete atrioventricular block are the two most common ways of bradyarrhythmia., ${ }^{1,2}$ If left untreated, the slow junctional escape rhythm is inadequate to support patients' hemodynamic needs. ${ }^{3}$ Currently, pacing with a pacemaker is the most effective treatment for patients with bradycardia and atrioventricular block. Right ventricular (RV) apex or RV side of the interventricular septum (IVS) is conventionally selected as the pacing site.

Correspondence to: Dr. Heng Zhang, Department of Cardiology, The First Affiliated Hospital of Bengbu Medical College, Bengbu, China

E-mail:724593321@qq.com

Received: July 26, 2021; Revised: September 14, 2021;

Accepted: October 12, 2021

DOI: https://doi.org/10.29271/jcpsp.2021.11.1268
However, such pacing strategies may cause intra-left ventricular (LV) and interventricular dyssychrony. Permanent Hisbundle pacing (HBP) is known as physiological pacing, which can synchronise the ventricles, improve cardiac function and reduce the risk of atrial fibrillation. ${ }^{4-7}$ The disadvantages of HBP include difficult implantation, large atrial signals complicating pacing management, the blocked site may be distal to the Hisbundle, and higher and unstable pacing threshold. ${ }^{8}$ Left bundle branch area pacing (LBBAP) has emerged as an alternative method to HBP. With the left bundle branch (LBB) spread over a relatively larger region and has a similar effect of synchronising the LV when being paced, LBBAP appears to be feasible and promising. ${ }^{9}$

The aim of the current study was to investigate the feasibility, safety, and effectiveness of LBBAP in patients with left bundle branch block (LBBB) morphology, right bundle branch block (RBBB) morphology and no bundle branch block (non-BBB) morphology. 


\section{METHODOLOGY}

Patients admitted to the First Affiliated Hospital of Bengbu Medical College for permanent pacemaker implantation were prospectively enrolled between May 2018 and January 2020. All patients met the criteria of treatment for bradycardia, based on the 2013 ESC/EHRA Guidelines. ${ }^{10}$ The study was approved by the Ethics Committee of Bengbu Medical College. Informed consents were obtained from all the subjects.

An $8 \mathrm{~F}$ peelable sheath was inserted first via left axillary vein or left subclavian vein. Under right anterior oblique view (RAO) $30^{\circ}$ fluoroscopic view, C315 sheath (Medtronic Inc., Minneapolis, MN USA) was inserted. After advancing it clockwise through the tricuspid valve and counterclockwise approaching the RV side of the IVS, the pacing lead (Model 3830, Medtronic Inc., Minneapolis, MN, USA) was advanced slightly beyond the distal part of the sheath to locate the His-bundle potential. Along the line between the located His potential and cardiac apex, the C315 sheath and pacing lead were moved 15 (10-20) mm towards the apex. Output of $5 \mathrm{~V} / 0.42 \mathrm{~ms}$ was applied and the "W" pattern of the paced QRS was viewed. The C 315 sheath was then turned counterclockwise with the head of the sheath appearing as concentric circles, and being fixed on the IVS. The perpendicular angle and depth of the insertion of the pacing lead was confirmed by left anterior oblique view (LAO) $45^{\circ}$ fluoroscopy. The end of the electrode was turned clockwise 4 to 5 times before pacing parameters were recorded and 12-lead ECG were analysed. The 3830 pacing lead was then further screwed clockwise into the LV side of the IVS. This process was closely monitored. Once the QRS morphology changed to RBBB morphology, or the duration of QRS and the stimulus to peak left ventricular activation time (Sti-LVAT) became reduced, the insertion of the lead was stopped. Corresponding pacing parameters and ECG characteristics were again recorded and analysed. The depth of the electrode into the IVS was confirmed by C315 sheath angiography (Figure 1). After LBBAP was confirmed, the pacemaker was connected for programming. ${ }^{11-13}$

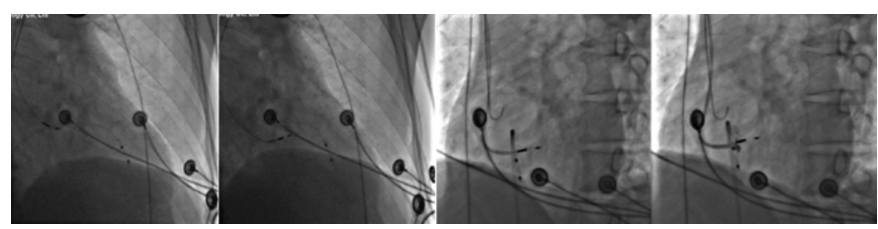

Figure 1: The location of pacing electrodes.

Under right anterior oblique view (RAO) $30^{\circ}$ fluoroscopic view, along the line between the located His potential and cardiac apex, the C315 sheath and pacing lead were moved 15 (10-20) $\mathrm{mm}$ towards the apex. The $\mathrm{C} 315$ sheath was then turned counterclockwise with the head of the sheath appearing as concentric circles, and being fixed on the IVS. The perpendicular angle and depth of the insertion of the pacing lead was confirmed by left anterior oblique view (LAO) $45^{\circ}$ fluoroscopy. The depth of the electrode into the IVS was confirmed by angiography.

There were two types of data that were collected. First were pacing related parameters, which included pacing threshold, pacing impedance and sensing amplitude. Secondly, the morphology of QRS complex on ECG was collected using a multichannel electrophysiological recorder (Jingjiang company, Sichuan, China) with a sweep speed at $100 \mathrm{~mm} / \mathrm{s}$. Paced QRS duration and Sti-LVAT were measured at V4-6. QRS duration was measured from the beginning of the $\mathrm{Q}$ wave to the end of the $\mathrm{S}$ wave. Sti-LVAT was measured as the time interval from the pacing stimulus to the line perpendicular to the peak of the $R$ wave in leads V4-V6.

The analysis was conducted using the SPSS software package (version 21, Chicago, Illinois, USA). Continuous variables were expressed as mean \pm standard deviation. Independent t-test or paired t-test were used to compare the difference between two independent groups or matched groups. One-way ANOVA was used for more than two group comparison with Tukey post hoc test for two-group comparisons. A p-value $<0.05$ was considered significant.

\section{RESULTS}

Twenty-one patients were included in the study, 15 men and 06 women. The mean age was $67.8 \pm 9.6$ (49 to 83 ) years. There were fifteen patients with essential hypertension, two with diabetes, eight with coronary heart disease, one with valvular disease and five with cerebral vascular disease. Prior to pacemaker implantation, LBBB morphology and RBBB morphology were found in five patients, respectively. Eleven patients had complete atrioventricular block, five had sick sinus syndrome, four patients had atrial fibrillation with long pauses, and one patient had heart failure with LBBB. Nineteen patients received a pacemaker implantation for the first time. The other two cases were admitted for pacemaker battery replacement. Two patients received VVI while DDD was applied in nineteen patients.

Table I: Comparing the pacing parameters and ECG characteristics between right and left sided IVS pacing.

\begin{tabular}{|c|c|c|c|c|}
\hline Parameters & RVP $(n=21)$ & LBBAP $(n=21)$ & $\mathbf{t}$ & $\mathbf{p}$ \\
\hline Pacing potential (V) & $0.8 \pm 0.2$ & $0.7 \pm 0.2$ & 1.313 & 0.204 \\
\hline Sensing amplitude (mV) & $8.3 \pm 3.9$ & $11.5 \pm 5.2$ & -2.308 & 0.032 \\
\hline Pacing impedance $(\Omega)$ & $727.8 \pm 150.5$ & $750.3 \pm 98.7$ & -0.587 & 0.564 \\
\hline Paced QRS duration (ms) & $168.9 \pm 14.1$ & $124.2 \pm 20.3$ & 9.699 & $<0.001$ \\
\hline Sti-LVAT (ms) & $119.1 \pm 12.2$ & $62.9 \pm 16.5$ & 14.177 & $<0.001$ \\
\hline
\end{tabular}

Typical changes in ECG were observed while advancing of pacing electrode from the right side of the IVS to the left side (under LV endocardium). When the pacing electrode was on right side of the IVS (RVSP), ECG morphology showed LBBB morphology. But when it was advanced to the left side of IVS (LBBAP), RBBB morphology appeared.

The paced QRS duration and the Sti-LVAT obtained when pacing on the right side of the IVS (RVSP) were significantly longer than the time intervals when pacing on left side of the IVS (LBBAP, Table I). As shown in Figures 2a-2c, such characteristic changes were observed in patients either with initial LBBB/RBBB morphology or non-BBB morphology. There was no significant 
difference in pacing threshold and impedance between LBBAP and RVSP, while the sensing amplitude was significantly higher when pacing from the LV side of the IVS (LBBAP).

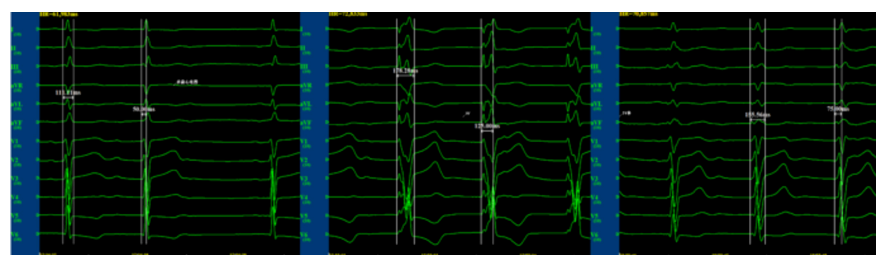

Figure 2-a: Characteristic ECG changes in a patient without bundle branch block morphology before LBBAP.

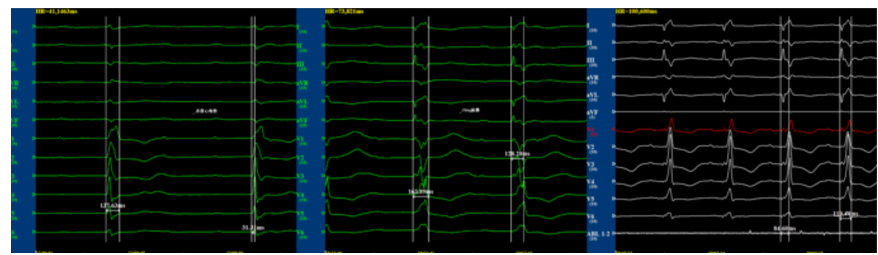

Figure 2-b: The characteristic change of ECG on patient with RBBB morphology before LBBAP.

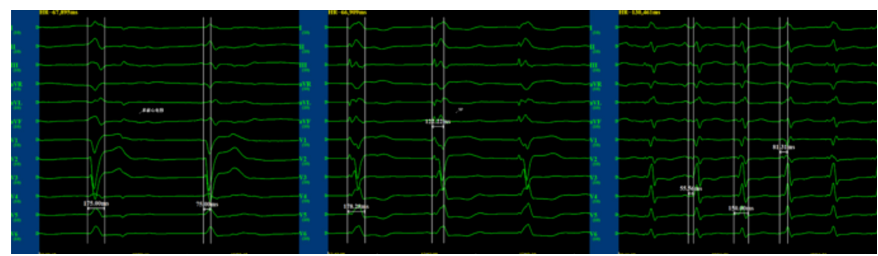

Figure 2-c: The characteristic change of ECG on patient with LBBB morphology before LBBAP.

Table II: Comparison between patients with LBBB morphology, RBBB morphology and non-BBB morphology.

\begin{tabular}{|c|c|c|c|c|c|}
\hline Parameters & $\begin{array}{c}\text { Without BBB } \\
(n=11)\end{array}$ & $\begin{array}{l}\text { LBBB } \\
(n=5)\end{array}$ & $\begin{array}{l}\text { RBBB } \\
(n=5)\end{array}$ & $\mathbf{F}$ & $\mathbf{p}$ \\
\hline $\begin{array}{l}\text { QRS duration before pacing } \\
\text { (ms) }\end{array}$ & $99.8 \pm 14.2 \# \Delta$ & $\begin{array}{l}174.6 \pm \\
10.9 * \#\end{array}$ & $137.4 \pm 15.9 * \Delta$ & 51.41 & $<0.001$ \\
\hline $\begin{array}{l}\text { QRS duration after pacing } \\
\text { (ms) }\end{array}$ & $126.7 \pm 23.2$ & $129.6 \pm 13.1$ & $113.2 \pm 18.6$ & 0.993 & 0.39 \\
\hline$\triangle \mathrm{QRS}$ duration $(\mathrm{ms})$ & $26.9 \pm 19.6 \# \Delta$ & $-45.0 \pm 19.7 \#$ & $-24.2 \pm 28.8 \Delta$ & 21.606 & $<0.001$ \\
\hline LVAT before pacing (ms) & $46.4 \pm 9.7 \#$ & $94.4 \pm 17.4^{*} \#$ & $43.4 \pm 9.7 *$ & 32.764 & $<0.001$ \\
\hline Sti-LVAT after pacing (ms) & $62.9 \pm 17.8$ & $63 \pm 15.9$ & $62.6 \pm 17.7$ & 0.001 & 0.999 \\
\hline$\triangle \mathrm{LVAT}(\mathrm{ms})$ & $\begin{array}{l}-16.6 \pm \\
15.0 \# \Delta\end{array}$ & $31.4 \pm 31.8^{*} \#$ & $-19.2 \pm 24.0 * \Delta$ & 9.502 & $<0.002$ \\
\hline
\end{tabular}

Table III: Changes in pacing parameters under LBBAP at follow-up (9.2 \pm 3.7 months).

\begin{tabular}{|l|c|c|c|c|}
\hline Parameters & $\begin{array}{c}\text { During the } \\
\text { operation }\end{array}$ & Follow-up & $\mathbf{t}$ & $\mathbf{p}$ \\
\hline $\begin{array}{l}\text { Pacing potential } \\
(\mathrm{V})\end{array}$ & $0.7 \pm 0.2$ & $0.9 \pm 0.4$ & -1.433 & 0.167 \\
\hline $\begin{array}{l}\text { Sensing amplitude } \\
(\mathrm{mV})\end{array}$ & $11.4 \pm 5.3$ & $9.5 \pm 3.4$ & 1.423 & 0.171 \\
\hline $\begin{array}{l}\text { Pacing impedance } \\
(\Omega)\end{array}$ & $750.3 \pm 98.7$ & $\begin{array}{c}695.7 \pm \\
113.3\end{array}$ & 1.559 & 0.135 \\
\hline
\end{tabular}

LBBAP: Left bundle branch pacing; VS: Interventricular septum.

An illustrative case showed, preoperatively, there was no bundle branch block morphology, with a QRS duration $111 \mathrm{~ms}$ and Sti-LVAT 50 ms on $V_{5}$ (on left panel). Pacing on the RV side of the IVS caused LBBB morphology, with a QRS duration $178 \mathrm{~ms}$ and Sti-LVAT 125 ms on $V_{5}$ (middle panel). Pacing on the LV side of the IVS caused RBBB morphology, with a QRS duration 156

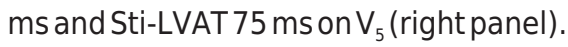

An illustrative case showed, preoperatively, there was complete RBBB morphology, with a QRS duration 138 ms and Sti-LVAT $31 \mathrm{~ms}$ on $\mathrm{V}_{5}$ (on left panel). Pacing on the RV side of the IVS caused LBBB morphology, with a QRS duration 164 ms and Sti-LVAT 128 ms on $V_{5}$ (middle panel). Pacing on the LV side of the IVS caused RBBB morphology, with a QRS duration $119 \mathrm{~ms}$ and Sti-LVAT $85 \mathrm{~ms}^{\circ} \mathrm{V}_{5}$ (right panel).

An illustrative case showed, preoperatively, there was complete LBBB morphology, with a QRS duration 175 ms and Sti-LVAT 72 ms on $V_{5}$ (on left panel). Pacing on the RV side of the IVS caused LBBB morphology, with a QRS duration 178 ms and Sti-LVAT 122 ms on $V_{5}$ (middle panel). Pacing on the LV side of the IVS caused RBBB morphology, with a QRS duration $150 \mathrm{~ms}$ and Sti-LVAT 81 ms on $V_{5}$ (right panel).

Patients initially with LBBB morphology showed significant reduction or a trend of reduction in paced QRS duration and StiLVAT compared to patients with RBBB morphology or non-BBB morphology (Table II). The reduction of paced QRS duration and Sti-LVAT were significantly greater in patients with RBBB morphology versus non-BBB morphology.

The pacemaker implantation was successful in all cases. There were no complications observed during the procedure, such as lead dislodgment, septal perforation, and coronary artery injury. At follow-up (9.2 \pm 3.7 months), the pacing parameters did not change significantly (Table III).

\section{DISCUSSION}

In the current study, 21 patients successfully performed pacing in the left bundle branch without complications, and one patient was changed to pacing the right ventricle septum due to the large right atrium, with a success rate of $95.5 \%$. Two patients of them underwent left bundle branch pacing and pacemaker replacement due to pacemaker energy depletion and increased impedance of the right ventricular electrode. There was a characteristic change of QRS morphology/duration and Sti-LVAT on ECG, while advancing the pacing lead through the IVS. Patients that initially had LBBB morphology in their intrinsic rhythm showed the greatest reduction in QRS duration and Sti-LVAT. Consistent with Chen's research, ${ }^{14}$ during the follow-up there were no adverse events observed and pacing parameters remained stable.

Based on the clinical guidelines, ${ }^{13,15}$ LBBAP can be confirmed by typical change of QRS morphology when advancing the pacing lead from the RV side to the LV side of the IVS which results in RBBB morphology; identification of the LBB potential; and shortened stimulus to LV activation time measured at the peak of the QRS waveform. However, LBB potential is recorded in only $50 \%$ to $80 \%$ of patients. ${ }^{16}$ Therefore, additional imaging methods are recommended for confirming LBBAP. In this study, angiography was used for confirming the LBBAP site.

This study indicates that both QRS duration and Sti-LVAT can be 
significantly shortened by LBBAP in patients with either intrinsic LBBB or RBBB morphology (Table II). Since LBBAP causes RBBB morphology, it is understandable that the overall benefit of correcting complete LBBB through pacing outweighs creating a RBBB morphology with shorter QRS duration. LBBAP could resynchronise the ventricles and improve cardiac function. ${ }^{17}$ Interestingly, LBBAP was shown to be beneficial in patients with complete RBBB morphology as well. Under LBBAP, the RBBB morphology was associated with shortened QRS duration and Sti-LVAT. Consistent with previous literature, this observation suggests there are fiber connections between the left and right bundle branches. ${ }^{14}$ This study also demonstrated an increase in QRS duration with LBBAP in patients with non-BBB morphology. However, these patients inevitably need pacemaker treatment. From our results compared to the RV sided IVS pacing, LBBAP was associated with shorter QRS duration. Prior studies have shown that LVBBP reduced the QRS duration to a greater extent than conventional RV pacing. ${ }^{14}$ Therefore, LVBBP may be suitable for patients both with and without bundle branch block. Among all cases, patients with LBBB morphology showed the greatest benefit with LBBAP, as they had the deepest reduction of QRS duration and LVAT.

This study has many limitations: This is a single-centre study. There were a limited number of cases included in the present study, and the patients enrolled represented mild disease. It would be interesting to recruit more patients with LBBB morphology and heartfailure as our study demonstrated significant improvement after LBBP in one patient with prior complete LBBB and LV dysfunction. The follow-up time after pacemaker implantation was short; therefore, long-term outcomes from LBBAP cannot be derived from the current study. Further, a randomised control group was not included. It was demonstrated that advancing the electrode from the right side of the IVS to its left side caused significant reductions in paced QRS duration and Sti-LVAT. The results of this study and results of others support the hypothesis that LVBBP is more physiological than conventional RV pacing. LV epicardial pacing or epicardial CRT are widely used to overcome ventricular dyssynchrony. The authors did not compare LBBAP with CRT in current study; however, LBBAP should show the same benefits since the paced patients of this study had reduced QRS duration. Future randomised control studies with longer followup duration are needed to fully elucidate the clinical benefits of LBBAP.

\section{CONCLUSION}

LBBP is feasible, appears to be safe, and may be promising as compared to the conventional RV pacing, it narrows the QRS duration. LBBP may be especially beneficial for patients with LBBB and heart failure. A randomised control study with larger sample size is needed.

\section{FUNDING:}

Bengbu Medical College (BYKY2019065ZD).
Ethical approval of this study was obtained from the Ethics Committee of First Affiliated Hospital of Bengbu Medical College, prior to initiation of the research work.

\section{PATIENTS' CONSENT:}

Informed consents were obtained from patients to publish the data concerning this study.

\section{CONFLICT OF INTEREST:}

The authors declared no conflict of interest.

\section{AUTHORS' CONTRIBUTION:}

TPC: Conception and design of the work; the acquisition, analysis, or interpretation of data for the work.

$\mathrm{XJS}, \mathrm{HZ}$ : Acquisition of data for the work; the acquisition, analysis, or interpretation of data for the work.

DYL: Acquisition of data for the work; drafting the work and final approval of the manuscript.

\section{REFERENCES}

1. Aguiar Rosa S, Timóteo AT, Ferreira L, Carvalho R, Oliveira $M$, Cunha $P$, et al. Complete atrioventricular block in acute coronary syndrome: Prevalence, characterisation and implication on outcome. Eur Heart J Acute Cardiovasc Care 2018; 7(3):218-23. doi: 10.1177/2048872617716387.

2. Adán V, Crown LA. Diagnosis and treatment of sick sinus syndrome. Am Fam Physician 2003; 67(8):1725-32.

3. Eliasson H, Sonesson SE, Salomonsson S, Skog A, WahrenHerlenius M, Gadler F, et al. Outcome in young patients with isolated complete atrioventricular block and permanent pacemaker treatment: A nationwide study of 127 patients. Heart Rhythm 2015; 12(11):2278-84. doi: 10.1016/j.hrthm.2015.06.028.

4. Dandamudi G, Vijayaraman P. How to perform permanent His bundle pacing in routine clinical practice. Heart Rhythm 2016; 13(6):1362-6. doi: 10.1016/j.hrthm.2016.03.040.

5. Lustgarten DL, Crespo EM, Arkhipova-Jenkins I, Lobel R, Winget $\mathrm{J}$, Koehler $\mathrm{J}$, et al. His-bundle pacing versus biventricular pacing in cardiac resynchronization therapy patients: A crossover design comparison. Heart Rhythm 2015; 12(7):1548-57. doi: 10.1016/j.hrthm.2015.03.048.

6. Sharma PS, Dandamudi G, Herweg B, Wilson D, Singh R, Naperkowski A, et al. Permanent His-bundle pacing as an alternative to biventricular pacing for cardiac resynchronisation therapy: A multicenter experience. Heart Rhythm 2018; 15(3):413-20. doi: 10.1016/j.hrthm.2017. 10.014 .

7. Huang W, Su L, Wu S, Xu L, Xiao F, Zhou X, et al. Long-term outcomes of His bundle pacing in patients with heart failure with left bundle branch block. Heart 2019; 105(2):137-43. doi: 10.1136/heartjnl-2018-313415.

8. Vijayaraman P, Dandamudi G, Worsnick S, Ellenbogen KA. Acute his-bundle injury current during permanent hisbundle pacing predicts excellent pacing outcomes. Pacing Clin Electrophysiol 2015; 38(5):540-6. doi: 10.1111/pace. 12571.

9. Huang W, Su L, Wu S, Xu L, Xiao F, Zhou X, et al. A novel pacing strategy with low and stable output: Pacing the left bundle branch immediately beyond the conduction block. 
Can J Cardiol 2017; 33(12):1736.e1-1736.e3. doi: 10.1016/ j.cjca.2017.09.013

10. Brignole $M$, Auricchio A, Baron-Esquivias G, Bordachar $P$, Boriani G, Breithardt OA, et al. 2013 ESC Guidelines on cardiac pacing and cardiac resynchronisation therapy: The task force on cardiac pacing and resynchronisation therapy of the european society of cardiology (ESC). Developed in collaboration with the european heart rhythm association (EHRA). Eur Heart J 2013; 34(29):2281-329. doi: 10.1093/ eurheartj/eht150.

11. Chen K, Li Y. How to implant left bundle branch pacing lead in routine clinical practice. J Cardiovasc Electrophysiol 2019; 30(11):2569-77. doi: 10.1111/jce.14190.

12. Cleland JG, Daubert JC, Erdmann E, Freemantle N, Gras D, Kappenberger $\mathrm{L}$, et al. The effect of cardiac resynchronisation on morbidity and mortality in heart failure. $N$ Eng/ J Med 2005; 352(15):1539-49. doi: 10.1056/NEJMoa050496.

13. Huang W, Chen X, Su L, Wu S, Xia X, Vijayaraman P. A beginner's guide to permanent left bundle branch pacing.
Heart Rhythm 2019; 16(12):1791-6. doi: 10.1016/j. hrthm.2019.06.016.

14. Chen K, Li Y, Dai Y, Sun Q, Luo B, Li C, et al. Comparison of electrocardiogram characteristics and pacing parameters between left bundle branch pacing and right ventricular pacing in patients receiving pacemaker therapy. Europace 2019; 21(4):673-80. doi: 10.1093/europace/euy252.

15. Chen X, Wu S, Su L, Su Y, Huang W. The characteristics of the electrocardiogram and the intracardiac electrogram in left bundle branch pacing. J Cardiovasc Electrophysiol 2019; 30(7):1096-1101. doi: 10.1111/jce.13956.

16. Zhang S, Zhou X, Gold MR. Left bundle branch pacing: JACC review topic of the week. J Am Coll Cardiol 2019; 74(24):3039-49. doi: 10.1016/j.jacc.2019.10.039.

17. Zhang W, Huang J, Qi Y, Wang F, Guo L, Shi X, et al. Cardiac resynchronisation therapy by left bundle branch area pacing in patients with heart failure and left bundle branch block. Heart Rhythm 2019; 16(12):1783-90. doi: 10.1016/ j.hrthm.2019.09.006. 